\title{
Angularly Uniform White Light-Emitting Diodes Using an Integrated Reflector Cup
}

\author{
L. Zhu, Student Member, IEEE, X. H. Wang, P. T. Lai, Senior Member, IEEE, and H. W. Choi, Senior Member, IEEE
}

\begin{abstract}
We report on white light-emitting diodes (LEDs) with a truncated-conical (TC) geometry produced by laser micromachining. A blue LED was shaped into a circular disc with $5^{\circ}{ }^{\circ}$-inclined sidewall using a modified laser micromachining setup. A layer of Al was coated onto the inclined sidewall and the bottom surface to form an integrated reflector. Due to the highly reflective mirror, laterally propagating photons are redirected into the upward direction, contributing to an increase of $21.7 \%$ of light intensity in the normal direction. With quantum dots applied to the surface, white light emission from this TC-LED structure demonstrated a $37 \%$ enhancement in color uniformity, compared with a conventional device.
\end{abstract}

Index Terms - Color uniformity, laser micromachining, light extraction, white light-emitting diode (LED).

\section{INTRODUCTION}

W HITE light-emitting diodes (LEDs) are set to replace conventional lighting technologies, such as incandescent bulbs and compact fluorescent lamps in the general illumination market [1], due to a number of distinct advantages including energy efficiency and being mercury-free amongst others. At present there are two main approaches for making white LEDs, including applying a yellow phosphor coating on a blue LED (or an ultraviolet LED with multiple phosphors), and color-mixing with discrete LED chips of red, green, and blue colors (RGB LED) [1]. The phosphor coating technique is widely adopted, although RGB LEDs are also employed in high-power applications such as for liquid crystal display (LCD) backlighting. With RGB LEDs, color-uniformity is an inherent disadvantage as the chips are spatially apart. For phosphor-coated LEDs, the placement and method of coating of phosphor(s) will also greatly influence the color uniformity, whereby emission homogeneity is an important attribute for many applications. This includes both angular uniformity from an individual LED and overall uniformity between the adjacent ones in an array.

In many cases, phosphor-coated white light LEDs are fabricated by applying an epoxy mixture containing phosphor particles onto the chip prior to encapsulation. The schematic diagram in Fig. 1(a) illustrates the coating profile of an LED coated in this way. The phosphor mixture is allowed to reflow in order to cover

Manuscript received December 03, 2009; revised December 23, 2009; accepted January 17, 2010. First published February 02, 2010; current version published March 10, 2010. This work was supported by a CERG grant of the Research Grant Council of Hong Kong (project HKU 7118/09E).

The authors are with the Department of Electrical and Electronic Engineering, The University of Hong Kong, Hong Kong (e-mail: hwchoi@hku.hk).

Color versions of one or more of the figures in this letter are available online at http://ieeexplore.ieee.org.

Digital Object Identifier 10.1109/LPT.2010.2041651
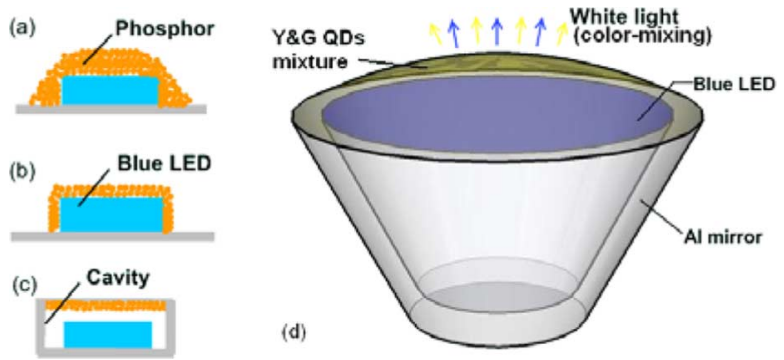

Fig. 1. Schematic diagram showing cross-sectional views of LED coating using three different methods: (a) phosphor-slurry coating; (b) conformal coating; (c) remote phosphor coating; (d) 3-D schematic diagram of a TC-LED (not to scale).

both the top and sidewalls, giving rise to a nonuniform distribution and thickness of coating, particularly at the edge of the chip. Such coating thickness nonuniformity, coupled with the fact that light emission from the top and sidewall are not of the same intensities, results in nonuniformity of color emission from different viewing angles [2]. This "halo effect" is well exemplified by the observation of a yellow ring at the periphery of the LED chip in Fig. 2(e). Alternative means of coating have been proposed to improve angular color uniformity. Thermal deposition or electrophoresis deposition ensures a more conformal phosphor layer across the planar and sidewall regions of the chip [3]. The method of remote phosphor, whereby the phosphor layer is located a certain distance away from the LED die, has also been proven to produce better color uniformity [4]. Schematic diagrams in Fig. 1(b) and (c) illustrate these two methods, respectively.

Rather than devising ways to improve coating uniformity on the device sidewall, the effect would certainly be more prominent if coating of the sidewall was no longer needed. This would require a complete redesign of the chip itself. We propose an LED chip with an integrated mirror reflector so that all the light is emitted exclusively through the top planar region. Laterally propagating modes would be redirected or reflected by the integrated mirror without being lost. This can be realized by shaping an LED chip into truncated cones (TCs) by laser micro-machining. Subsequently, a reflective metallic layer was deposited on the bottom and slanted sidewall surfaces of the chip. Finally, a color converter can be coated onto the top planar window. A 3-D schematic diagram of this novel chip design can be found in Fig. 1(d).

This concept originates from LED packages with a mirrorcoated cavity, which has been proven to alleviate issues with color-mixing. Distinguishing from that, our reflector cup has been integrated right onto the LED, offering a solution at the root of the problem, which should be able to eliminate the "halo 


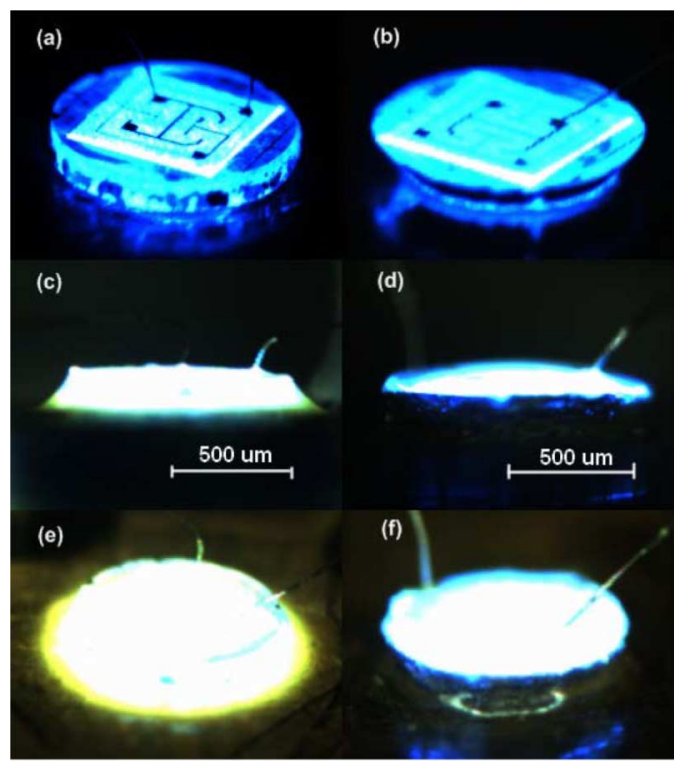

Fig. 2. Operational images of the packaged devices: (a) and (b) are the circular reference LED and TC-LED; (c) and (e) show the QD-coated circular reference LED, while (d) and (f) show the QD-coated TC-LED.

effect" as emission through the sidewalls is completely suppressed. The optical characteristics and performance of this redesigned white LED is evaluated and compared with that of a conventional design.

\section{EXPERIMENTAL DETAILS}

The excitation blue InGaN-GaN LED was fabricated by standard microfabrication techniques, details of which can be found in references such as [5]. The wafers were then thinned down to $\sim 150 \mu \mathrm{m}$. The process begins to differ from the conventional flow at the step of wafer dicing. Although laser-micromachining is commonly adopted for die separation, our setup is modified to enable machining of oblique sidewalls [6]. A UV pulsed laser at $349 \mathrm{~nm}$ serves as the source. After collimation and expansion, the beam was reflected and focused using a triplet lens onto a sample placed on the focal plane. A laser turning mirror was inserted between the focusing optics and the sample, causing the beam to strike the sample at any arbitrary oblique angle thus enabling oblique sidewalls to be formed. At the same time, a rotary stage allows the chips to be diced into circular forms with diameters of $800 \mu \mathrm{m}$ on the emission face, giving the LEDs truncated conical geometries. LEDs of circular geometry have been demonstrated to offer enhancement of light extraction and emission of axial symmetry [6]. As determined from our previous work, an inclination angle of $50^{\circ}$ serves its purpose best and thus the inverted truncated-cone LED (TC-LED) is shaped with a $50^{\circ}$ inclined sidewall [7]. For reference, an identical circular chip was prepared, except that the sidewalls were vertical.

After chip separation, a 500-nm-thick Al layer was deposited both onto the inclined sidewall and the bottom surface of the TC-LED by e-beam evaporation. This forms the reflector cup with a highly reflective metallic mirror, preventing photons from escaping from the sidewall and yet redirecting them upwards towards the top emission window. In this way, photons propagating sideways (the guiding mode photons) are never wasted. The operational images of this LED, together with the reference LED, are shown in Fig. 2(a) and (b).

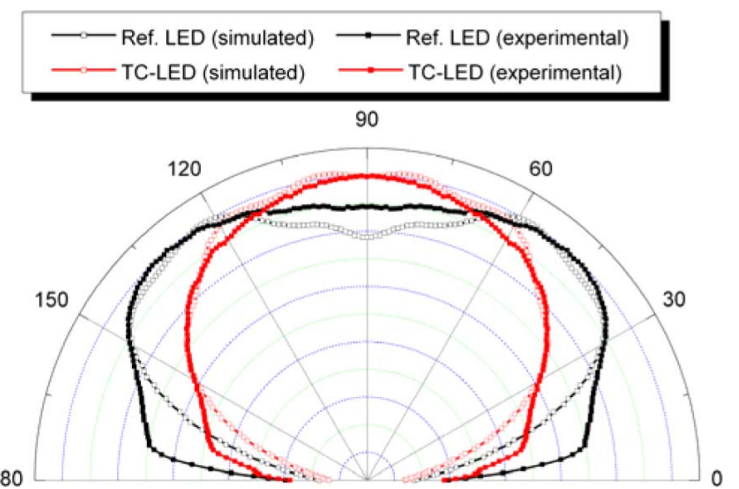

Fig. 3. Experimental and simulated emission patterns of the devices.

Quantum dots (QDs) are used in this work as color-conversion agents. Green $(540 \mathrm{~nm})$ and yellow $(560 \mathrm{~nm})$ light-emitting QDs (Evident Technologies) are mixed together to a volume ratio of $7: 5$ for balanced white light emission. The mixture is then blended with transparent UV epoxy (Norland 61), following which a small volume of the slurry is dispensed onto the chips. The chips have been packaged into standard TO-cans. There are several factors that can influence the color uniformity of an LED, including accurate control of the concentration of QDs, particle settling both before and after dispensing, and the distribution of the mixture around the die. To minimize these effects, the epoxy was spin-coated onto the chips to ensure evenness of the coating. The completed devices were tested at a bias current of $20 \mathrm{~mA}$. Fig. 2(c) and (e) illustrates emission from the reference LED structure from two different angles. A ring of yellow light is clearly observed at the periphery of the circular chip, attributed to the relatively thicker coating on the sidewall. This effect is evidently suppressed with the TC-LED structure as shown in Fig. 2(d) and (f), whereby uniform white light emission is observable from different angles. This can be attributed to the integrated reflector cup on the chip. To systematically assess the angular uniformity, the far-field radiation patterns of the devices were measured.

\section{RESULTS AND DISCUSSION}

The optical characteristics of the TC-LEDs were evaluated and compared with conventional LEDs with normal sidewall facets. The angular emission patterns of the devices were obtained by rotating a fiber probe coupled to a spectrometer about their central axis in the range of $-90^{\circ}$ to $90^{\circ}$, in steps of $1^{\circ}$. As observed from Fig. 3, the divergence half-angle $\gamma$ of the TC-LED is $53^{\circ}, 26^{\circ}$ less than that of reference LED with $\gamma$ of $79^{\circ}(\gamma$ is defined as the angle at which power intensity drops to $2^{1 / 2}$ of its peak value). The modified emission pattern also becomes almost perfectly lambertian. The overall optical intensity of the TC-LED, obtained by integrating the angular intensities over $180^{\circ}$, is found to be $15.8 \%$ lower than that of the reference LED. Nevertheless, if we consider emission in the direction normal to the device a pronounced increase in optical intensity of $16.7 \%$ is evaluated. The component of light emitted in the normal direction from an LED is important and useful as it can readily be collected with external optics, while photons emitted at large divergence angles will simply be lost. The increase in emission in the normal direction is attributed to redirection of laterally propagating photons via the integrated reflector cup. 


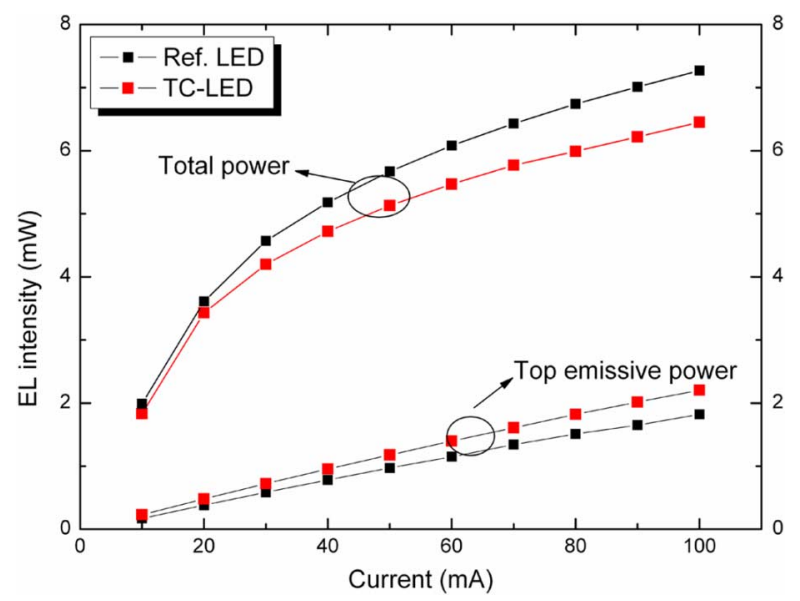

Fig. 4. $L-I$ curve comparing the performances of TC-LED and the reference LED.

The result is verified with ray-tracing simulation using Trace Pro; the simulated emission profiles are plotted together with the experimental data in Fig. 3. The simulated plot is consistent with the experimental plot: the TC-LED exhibited a $16^{\circ}$ reduction of divergence half-angle, a $15.8 \%$ decrease of overall intensity, and a $21.8 \%$ increase of intensity in the normal direction. Deviation between simulated and experimental data occurs at larger divergence angles, which may be explained in terms of the roughened sidewalls processed by laser micromachining.

Fig. 4 shows the light output-current $(L-I)$ plots for the TC-LED and reference LED. The total output powers were measured by placing the packaged devices within a 12-in integrating sphere, which is fiber-coupled to a calibrated optical spectrometer. According to the data in Fig. 4, the total optical power of the TC-LED dropped by $10.9 \%$ on average compared with the reference LED. Although the photons are redirected by the mirror-coated sidewall, some of these photons are reabsorbed in the $\mathrm{GaN}$ layers, giving rise to an overall reduction of total emission. On the other hand, the emission power in the normal direction was measured with a collection fiber (400- $\mu \mathrm{m}$ diameter) placed $1 \mathrm{~cm}$ away from the device. Under this measurement geometry, the optical power of the TC-LED was enhanced by $21.7 \%$ on average compared with the reference LED.

The color uniformity of our devices was determined in a systematic way through angle-resolved measurements of color coordinates based on the CIE 1931 color space system, which is widely adopted for assessing color quality of general lighting sources [8], [9]. The experimental setup is identical to that of emission pattern measurement. In this case, the CIE $x, y$ coordinates were evaluated from the measured spectrum as a function of angle and plotted in Fig. 5. The angular color uniformity is defined as the ratio of the maximum and minimum magnitudes within the measurement range. As indicated in Fig. 5, the device without an integrated reflector produced a wide variation in CIE coordinates, with maximum deviations of 0.0627 $(\Delta x)$ and $0.0601(\Delta y)$, thus resulting in a mediocre color uniformity figure of 0.542 . On the other hand, the angular pattern of the TC-LED appears flatter, exhibiting reduced deviations over the measurement range, with values of $0.0285(\Delta x)$ and 0.0309 $(\Delta y)$, respectively. Correspondingly, it provided a more satisfying color uniformity factor of 0.745 , a remarkable improve-

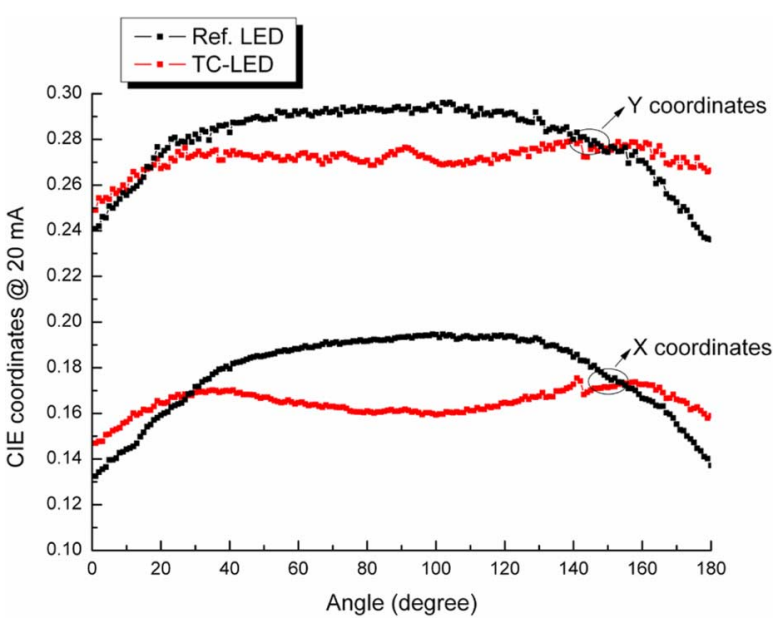

Fig. 5. Experimentally measured angular-resolved CIE coordinates of the QD-coated TC-LED and reference LED.

ment of $37 \%$ over the conventional device. The results support our claim of achieving improved color uniformity with the proposed LED design with an integrated reflector cup, although the luminous efficacy was slightly compromised, evaluated to be 97.6 and $115.1 \mathrm{~lm} / \mathrm{W}$ for the TC-LED and reference LED at $20 \mathrm{~mA}$, respectively.

\section{CONCLUSiON}

With a specially designed chip structure produced by laser micro-machining, the TC-LED with an integrated reflector demonstrated higher emissive power in the normal direction and superior angular color uniformity, making it highly suited for general lighting applications. The improvements are attributed to the reflective mirror coated onto the inclined sidewall. It prevents photons from being emitted laterally through the sidewalls and redirecting them into the escape cone, resulting in a $21.7 \%$ enhancement of top emissive power.

\section{REFERENCES}

[1] E. F. Schubert and J. K. Kim, "Solid-state light sources getting smart," Science, vol. 308, pp. 1274-1278, 2005.

[2] F. K. Yam and Z. Hassan, "Innovative advances in LED technology," Microelectron. J., vol. 36, pp. 129-137, 2005.

[3] J. B. Talbot, E. Sluzky, and S. K. Kurinec, "Electrophoretic deposition of monochrome and color phosphor screens for information displays," J. Mater. Sci., vol. 39, pp. 711-778, 2004.

[4] Narendran, "Improved performance white LED," in Proc. SPIE, Bellingham, WA, vol. 5941, pp. 45-50.

[5] K. N. Hui, W. Y. Fu, W. N. Ng, C. H. Leung, P. T. Lai, K. K. Y. Wong, and H. W. Choi, "Polychromatic light-emitting diodes with fluorescent nanosphere opal coating," Nanotechnology, vol. 19, 2008, Article 355203

[6] X. H. Wang, W. Y. Fu, P. T. Lai, and H. W. Choi, "Evaluation of InGaN/GaN light-emitting diodes of circular geometry," Opt. Express, vol. 17, pp. 22311-22319, 2009, 25.

[7] W. Y. Fu, K. N. Hui, X. H. Wang, P. T. Lai, and H. W. Choi, "Geometrical shaping of InGaN light-emitting diodes by laser micromachining,' IEEE Photon. Technol. Lett., vol. 21, no. 15, pp. 1078-1080, Aug. 1, 2009.

[8] D. A. Steigerwald, J. C. Bhat, D. Collins, R. M. Fletcher, M. O. Holcomb, M. J. Ludowise, P. S. Martin, and S. L. Rudaz, "Illumination with solid state lighting technology," IEEE J. Sel. Topics Quantum Electron., vol. 8, no. 2, pp. 310-320, Mar./Apr. 2002.

[9] S. Muthu, F. J. P. Schuurmans, and M. D. Pashley, "Red, green, and blue LEDs for white light illumination," IEEE J. Sel. Topics Quantum Electron., vol. 8, no. 2, pp. 333-338, Mar./Apr. 2002. 